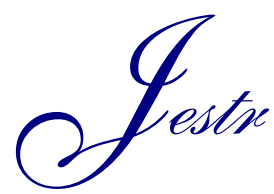

Research Article

\title{
Receiver Intend to Reduce Ambient Light Noise in Visible-Light Communication using Solar Panels
}

\author{
K. Sindhubala* and B.Vijayalakshmi
}

ECE Department, B. S. Abdur Rahman University, Chennai, India

Received 13 July 2016; Accepted 11 February 2017

\begin{abstract}
In the recent years, optical wireless communication using light emitting diode (LED) has increased lot of interest among researchers popularly known as visible-light communication (VLC). In such VLC system, an optical sensor used in the receiver side converts the modulated light signal into electrical signal. Conventionally, photo-diode (PD) is used as an optical sensor to serve this purpose. Instead of photo-diode, solar panel can be used as an optical sensor as it has the advantages of large collection area, easier targeting of light from an optical source, requires no external power supply and it shuns the reqirement of trans-impedance amplifier (TIA). However, ambient light noise due to indirect sunlight and fluorescent light is a major concern that affects the performance of the communication system in an indoor environment. In this paper, a new design of VLC receiver to reduce ambient light noise using solar panels is proposed. Experimental results show that the proposed receiver is capable of reducing ambient light noise caused by indirect sunlight and fluorescent lamp in an indoor environment. The implemented VLC system is capable of operating at $5.78 \mathrm{kHz}$ and transmission distance of $0.40 \mathrm{~m}$ is achieved.
\end{abstract}

Keywords: Ambient light noise, fluorescent light, indirect sunlight, visible-light communication (VLC), VLC receiver.

\section{Introduction}

The optical wireless communication using LED for both illumination and communication is called as visible-light communication (VLC). VLC technologies are considered as a supplement technology for RF communication because of the advantages such as huge amount of unregulated bandwidth, communication security, hazardless to humans and license-free [1-6]. VLC system usually consists of light emitting diode (LED) and photo-diode (PD) as the optical front-end devices.PIN PD or avalanche PD is used as an optical sensor in the conventional VLC system with a suitable biasing circuitry and trans-impedance amplifier (TIA) [7-8]. Instead of PD, solar panel can be used as an optical sensor as it has the advantages of large collection area, easier targeting of light from an optical source, requires no external power supply and it eliminates the need of transimpedance amplifier (TIA) [9 -16]. In this proposed work, commercially available light emitting diode (LED) and solar panel are used for data transmission and reception. The performance of the VLC system is affected by the ambient light noise inside the indoor environment. The received optical signal at the solar panel contain modulated input signal, DC component from indirect sunlight and rectified sine wave form from fluorescent light generated by conventional ballast. The natural ambient light noise from indirect sunlight changes with respect to time in an indoor environment.

E-mail address: sindhubala_ece_phd_2014@bsauniv.ac.in

ISSN: $1791-2377$ @ 2017 Eastern Macedonia and Thrace Institute of Technology. All rights reserved.
Few research works are reported to address the undesirable effect of ambient light noise in VLC system using solar panel as receiver. A capacitor to filter DC component and conventional low pass filter to filter out the other sources of noise is reported $[9,10]$. Using low pass filter, ambient light noise cannot be eliminated completely. It also reports solar panel receiver can be used for both data reception and simultaneous energy harvesting. VLC signal is retrieved from the sunlight interference by subtracting $\mathrm{V}_{\max }$ and $V_{\min }[11]$. However, no dedicated experimental design to reduce the ambient light noise from fluorescent lamp and to retrieve back the original signal is reported. The DC component due to external radiation caused by sunlight is eliminated using capacitor [13]. The ambient noise caused due to other external lightning is also a major concern that affects the performance of the system, however using the above method only natural ambient light noise can be eliminated, not the artificial ambient light noise.

The proposed work investigates the effects of natural and artificial ambient light noise using solar panel as receiver in VLC system. The receiver structure designed must be simple, low cost and it must reduce the ambient light noise. The proposed receiver consists of two solar panels arranged in such a way solar panel ${ }_{1}$ collects the LED signal and ambient light and solar panel ${ }_{2}$ placed on the opposite side at angle of $180^{\circ}$ receives only the ambient light. The arrangement must be such that both the solar panels track the equal amount of ambient light. Difference amplifier is used in the next stage to reduce the ambient light noise and recover back the received signal with the reduction of ambient light noise. Results show that the proposed receiver effectively reduces the ambient light noise from indirect 
sunlight and fluorescent lamp generated by conventional ballast.

The research paper is ordered as the following sections; section 2 describes the system design of VLC system using the proposed receiver. In section 3 , the testing and the results of the proposed VLC system are discussed. Finally, section 4 concludes the paper.

\section{System design of VLC system using the proposed receiver}

VLC system mainly consists of transmitter and receiver. Line of sight (LOS) channel model is considered between the transmitter and receiver.The overall block diagram of VLC system using the proposed receiver is shown in Fig.1.

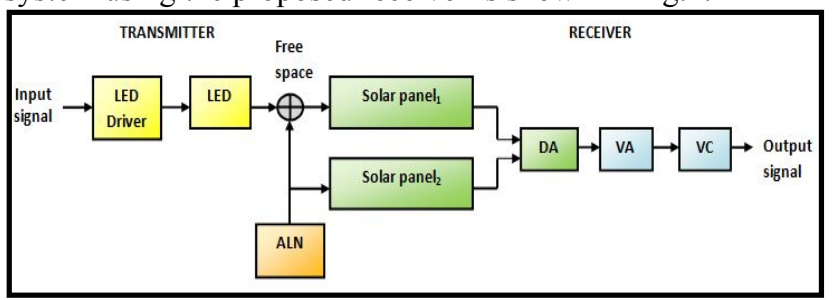

Fig.1.Block diagram of VLC system using the proposed receiver ALN: Ambient light noise DA: Difference amplifier Voltage amplifier

VC: Voltage comparator

\subsection{Transmitter design}

Transmitter of the proposed VLC system uses On-Off keying (OOK) modulation because of the simplicity and reduced power consumption compared to other modulation schemes. LM555 timer is used to produce the OOK signals of 5.78 $\mathrm{kHz}$. The LED driver takes the input signal and drives the white LED. The LED driver consisting of resistor prevents the high current flowing through the white LED. Implemented transmitter uses $1 \mathrm{~W}$ high power white LED of forward current $0.35 \mathrm{~A}$ and viewing angle $90^{\circ}$ and light intensity of 80 Lumens. Thus the transmitter converts the input electrical signal into variations of light intensity. Fig. 2.shows the circuit diagram of the proposed VLC transmitter.

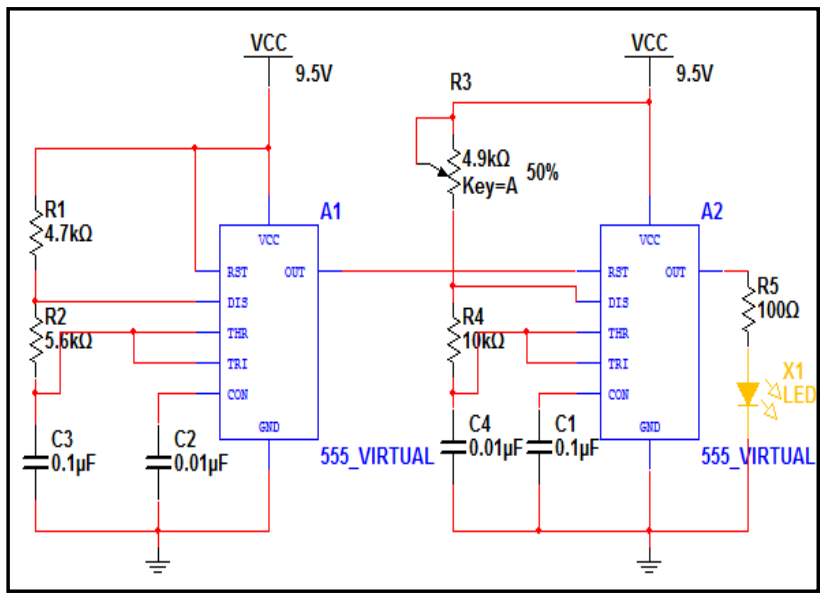

Fig.2. Circuit diagram of proposed VLC transmitter

\section{2.Line of sight (LOS) channel}

In this proposed work, the LOS wireless channel is considered between the transmitter and receiver. LOS Channel DC gain for the LED source [15] is given by
$H(0)=\left\{\begin{array}{cr}\frac{\mathrm{A}(\mathrm{m}+1)}{2 \pi \mathrm{d}^{2}} \cos ^{\mathrm{m}}(\varnothing) \cos (\psi), & 0 \leq \psi \leq \Psi_{\mathrm{c}} \\ 0 & \psi>\psi_{\mathrm{c}}\end{array}\right\}$

Where $\mathrm{d}$ is the distance between the transmitter and receiver, $\psi$ is the angle of incidence with respect to the receiver axis, $\psi_{\mathrm{c}}$ is the FOV of the receiver.

The received power is given by

$\mathrm{p}_{\mathrm{r}}=\mathrm{H}(0) * \mathrm{p}_{\mathrm{i}}$

$\mathrm{H}(0)$ is the DC channel gain and $\mathrm{p}_{\mathrm{i}}$ is the transmitted optical power of LED source.

\section{3. Receiver design}

\section{3.1.Solar panel characteristics}

The solar panel characteristics are experimented first to test whether the solar panel can act as a detector for proposed VLC system. The solar panel used is the JIL-ECO 003, multi crystalline silicon solar panel with rectangular shape of size $250 \times 145 \times 15 \mathrm{~mm}$, module efficiency of $10 \%$ manufactured by Frontech Company. The open circuit voltage of solar panel is $10.7 \mathrm{~V}$ and its short circuit current is $0.38 \mathrm{~A}$. Fig.3.shows the V-I characteristics of solar panel for different optical power from white LED. The electrical power of solar panel is obtained by multiplying the voltage and current. The incident optical power for the first case is $0.38 \mathrm{~mW}$, the maximum electrical power obtained from the solar panel is $0.04 \mathrm{~mW}$ at the load resistance of $60 \mathrm{~K} \Omega$. Thus the optical to electrical conversion efficiency obtained is $9.5 \%$. The incident optical power for the second case is $0.69 \mathrm{~mW}$. The maximum electrical power obtained from the solar panel is $0.07 \mathrm{~mW}$ at the load resistance of $70 \mathrm{~K} \Omega$. Thus the optical to electrical conversion efficiency obtained is $9.8 \%$. The incident optical power for the third case is $1.22 \mathrm{~mW}$. The maximum electrical power obtained from the solar panel is $0.12 \mathrm{~mW}$ at the load resistance of $30 \mathrm{~K} \Omega$. Thus the optical to electrical conversion efficiency obtained is $9.83 \%$.

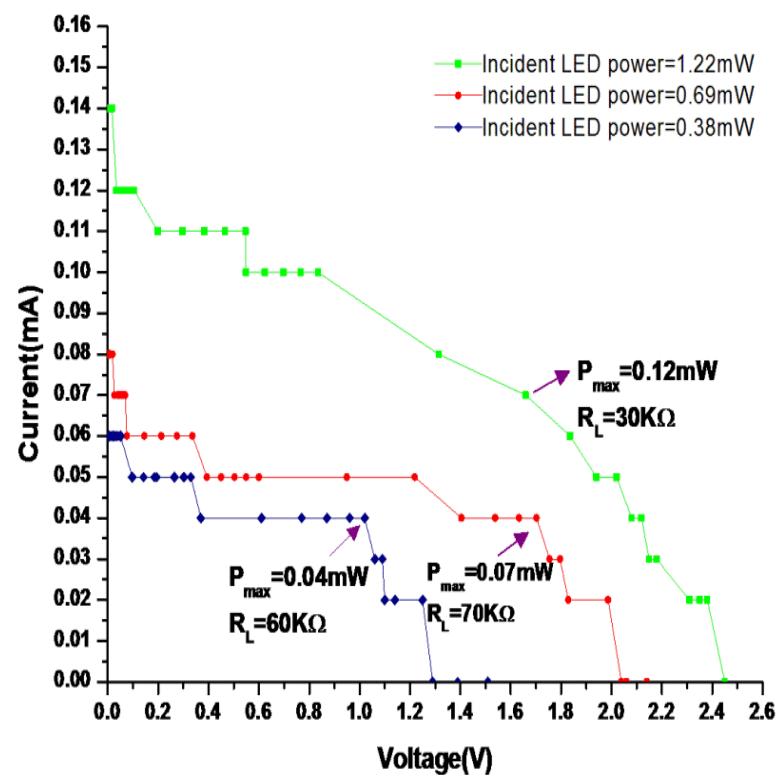

Fig.3.V-I characteristics of solar panel for different optical power from white LED

The electrical power-current characteristics of solar panel for different optical power from white LED are illustrated in Fig.4. 


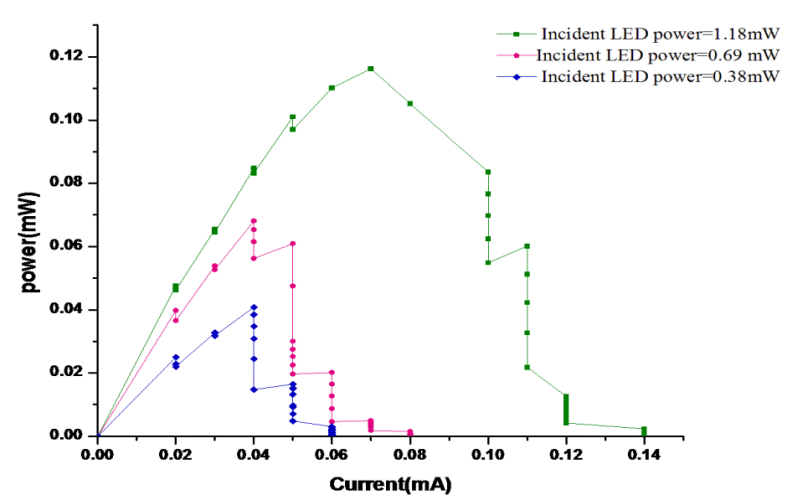

Fig. 4.P-I characteristics of solar panel for different optical power from white LED

Fig.5. shows the measured frequency response of solar panel for different voltages across the white LED. In Fig.5, it can be observed that the maximum achievable bandwidth of solar panel is $10 \mathrm{kHz}$ and the solar panel can receive even low frequency signals.

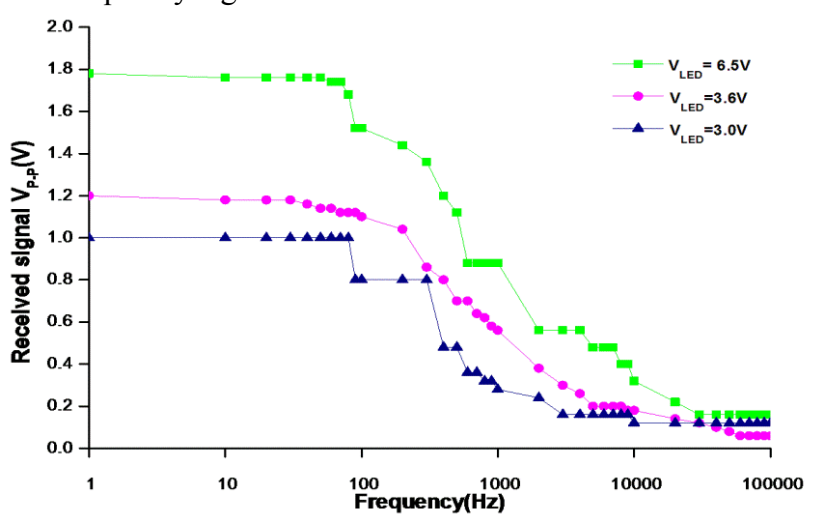

Fig.5. Frequency response of solar panel for different voltage across white LED.

The proposed VLC receiver design consists of two commercially available solar panels.

- Solar panel $_{1}$ : for detecting LED signal and ambient light noise

- Solar panel $_{2}$ :detecting the ambient light noise

The arrangement of solarpanel $l_{1}$ and solar panel $_{2}$ are kept in such a way no transmitted LED light falls on solarpanel ${ }_{2}$. The two solar panels are kept at an angle of $180^{\circ}$. The two solar panels are first tested whether it can detect equal amount of ambient light by switching off the transmitted LED signal. In the electronics lab of B.S.Abdur Rahman University where the experimentation is carried out solar panel $_{1}$ tracked more ambient light than solar panel ${ }_{2}$. The two solar panels ambient light voltage levels must be same then only the ambient light noise can be attenuated easily. Hence a voltage source $\mathrm{V}_{\mathrm{s}}$ is added to the output of solarpanel $\mathrm{l}_{2}$ to make both the solar panels track equal ambient light noise. Then, the experimentation is carried out by switching on the transmitted signal through LED. Thus solar panels convert the received optical signal into electrical signal. Difference amplifier is used in the next stage to mitigate the ambient noise. The received signal is weak at this stage. Hence, voltage amplifier is used to amplify the signal. Finally the digital signal is reconstructed back using voltage comparator.
Difference amplifier, voltage amplifier and voltage comparator is implemented using LM324 quadruple operational amplifier. LM324 is chosen due to four op-amps in a single package, wide bandwidth of $1 \mathrm{MHz}$ and large DC voltage gain of $100 \mathrm{~dB}$. The circuit diagram of proposed VLC receiver using solar panels is depicted in Fig.6.

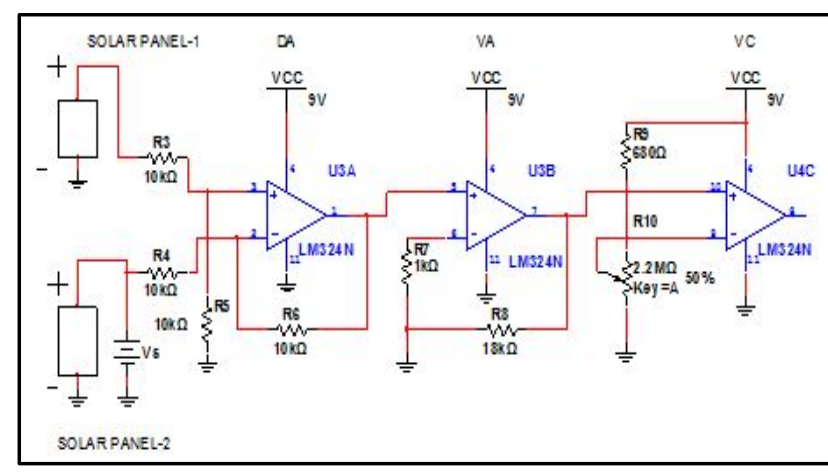

Fig.6. Circuit diagram of proposed VLC receiver using solar panels employing ambient light noise reduction technique

\subsubsection{Mathematical analysis}

The total voltage produced at solarpanel $_{1}\left(\right.$ vout $\left.1_{1}\right)$ is given by the eq (3)

$$
\mathrm{V}_{\text {out } 1}=\mathrm{V}_{\mathrm{s}}+\mathrm{V}_{\mathrm{n}}
$$

Where $\mathrm{V}_{\mathrm{S}}$ the voltage is produced due to LED signal and $\mathrm{V}_{\mathrm{n}}$ is the voltage produced due to ambient light noise sources from indirect sunlight and fluorescent lamp.

The voltage produced at solarpanel $\mathrm{l}_{2}$ (vout2) is given by the eq (4)

$$
\mathrm{V}_{\text {out2 }}=\mathrm{V}_{\mathrm{n}}
$$

Where $\mathrm{V}_{\mathrm{n}}$ the voltage is produced due to ambient light noise sources from indirect sunlight and fluorescent lamp.

The difference amplifier (DA) is used to attenuate the ambient light noise from the received signal. The output voltage produced at the difference amplifier stage is given by eq (5)

$$
\begin{aligned}
V_{\text {DA }}= & V_{\text {out } 1}-V_{\text {out } 2} \\
= & V_{\mathrm{s}}+V_{\mathrm{n}}-V_{\mathrm{n}} \\
& =\mathrm{V}_{\mathrm{s}}
\end{aligned}
$$

The signal received in the difference amplifier (DA) stage is very weak, hence voltage amplifier (VA) is used to amplify the signal. The output voltage produced at the voltage amplifier stage is given by eq (8)

$$
V_{v A}=A v *\left(V_{D A}\right)
$$

Where $\mathrm{A}_{\mathrm{V}}$ the voltage is gain and $\mathrm{V}_{\mathrm{DA}}$ is the voltage produced at the DA stage.

Voltage comparator is used to recover back the transmitted OOK signals with the mitigation of ambient light noise in indoor environment. 


\section{Experimental results and discussion}

The experimentation is carried out inside the electronics laboratory of B.S Abdur Rahman University. The ambient light sources inside the room include indirect sunlight coming through the windows and fluorescent light driven by conventional ballast. Communication distance of $0.40 \mathrm{~m}$ is considered between the transmitter and receiver. The results are discussed in the subsequent section

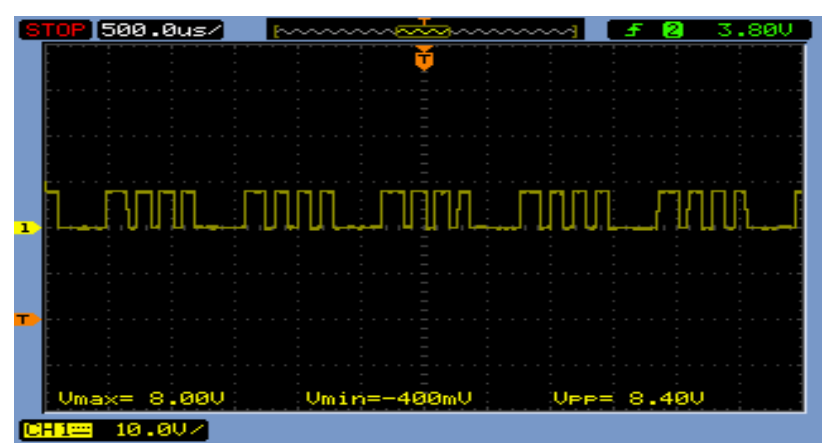

Fig.7a.Time-domain waveform of the input

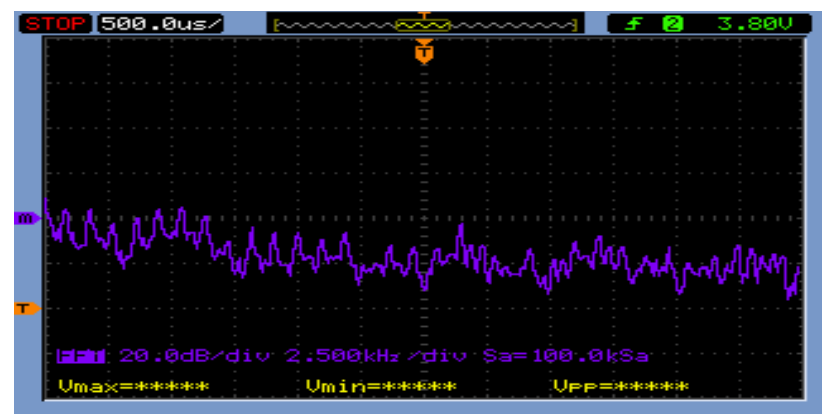

Fig.7b.spectrum of the input signal signal

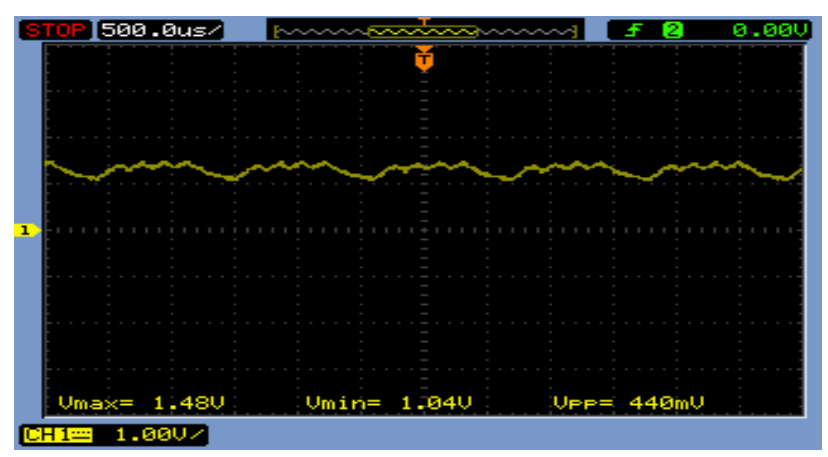

Fig.8a.Time-domain waveform of the

solar panel ${ }_{1}$ signal

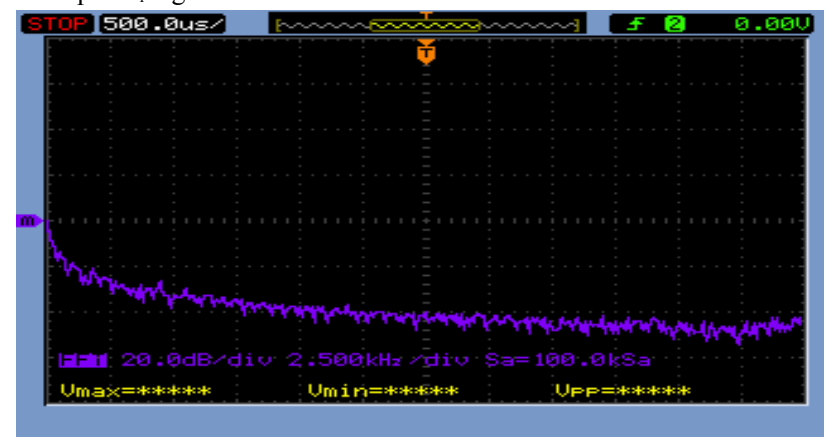

Fig.8b.Spectrum of the solar panel signal

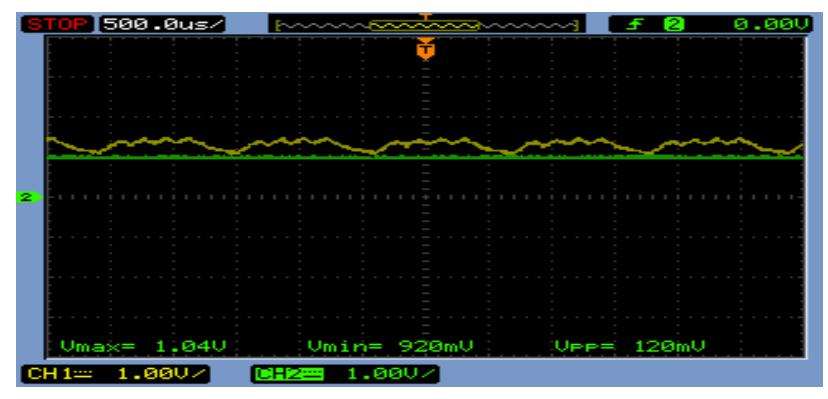

Fig.9a.Time-domain waveform of the solar panel $_{2}$ signal

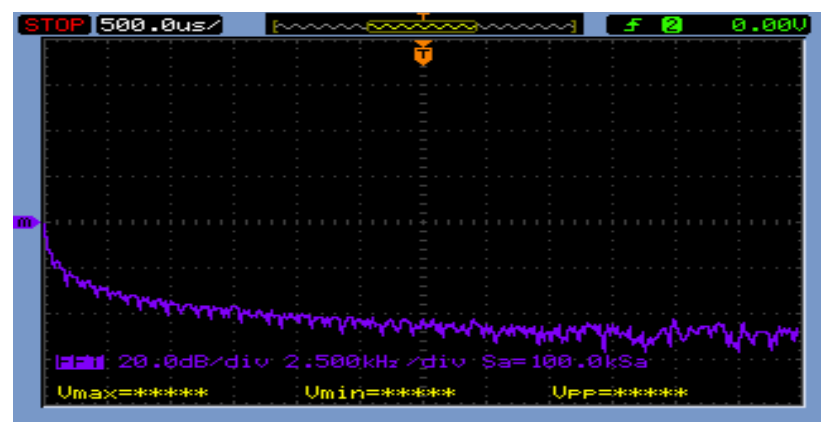

Fig.9b.Spectrum of the solar panel $_{2}$ signal

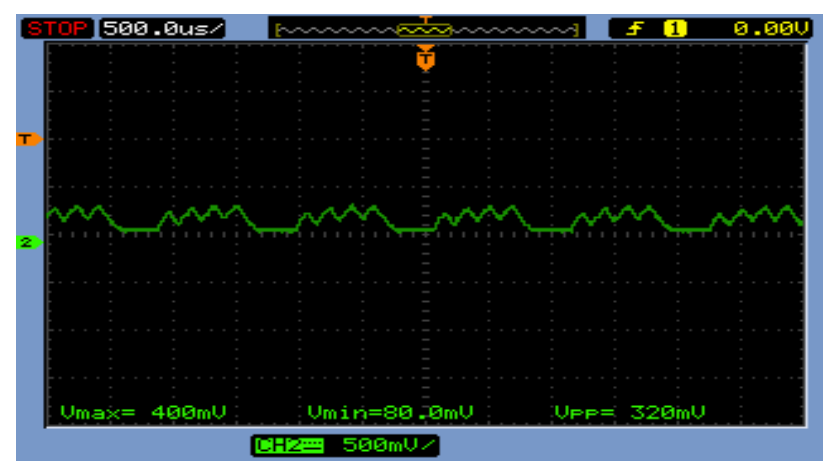

Fig.10a.Time-domain waveform of the amplifier (DA) signal

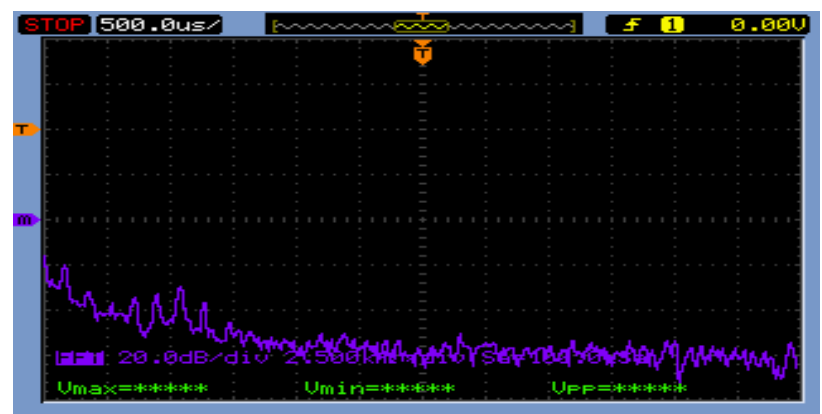

Fig.10b.Spectrum of the difference difference amplifier(DA) signal

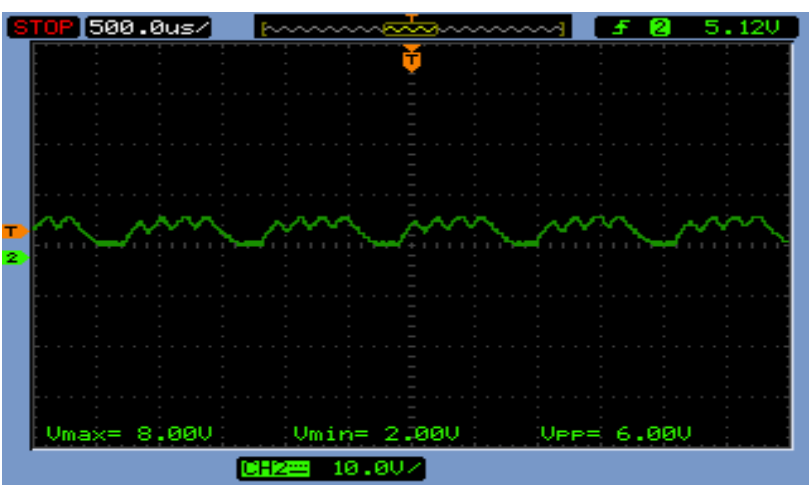

Fig.11a.Time-domain waveform of the voltage amplifier (VA) signal 


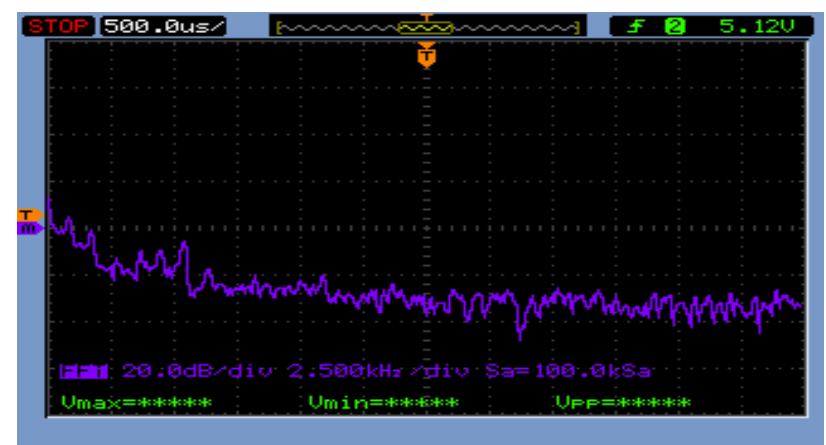

Fig.11b.Spectrum of the voltage amplifier (VA) signal

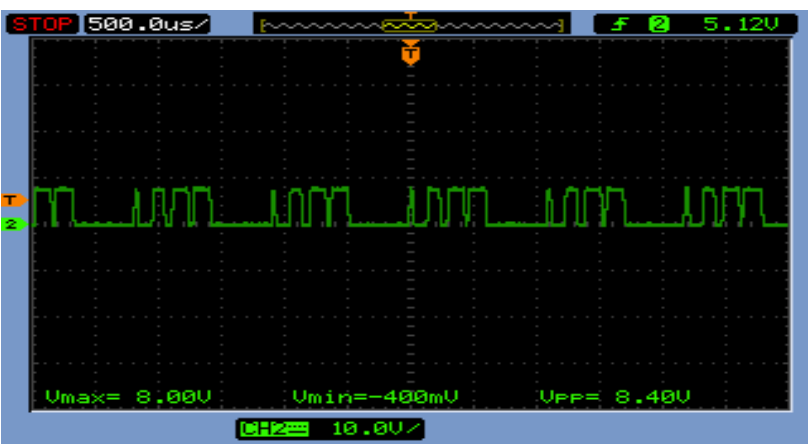

Fig.12a.Time-domain waveform of the voltage comparator (VC) signal

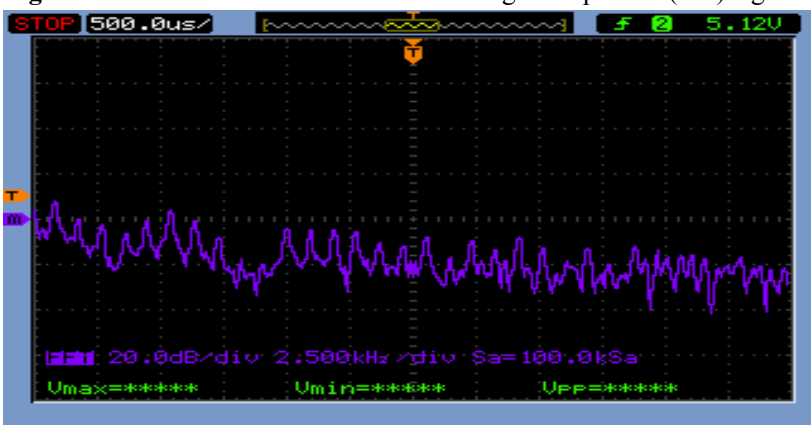

Fig.12b.Spectrum of the voltage comparator (VC) signal

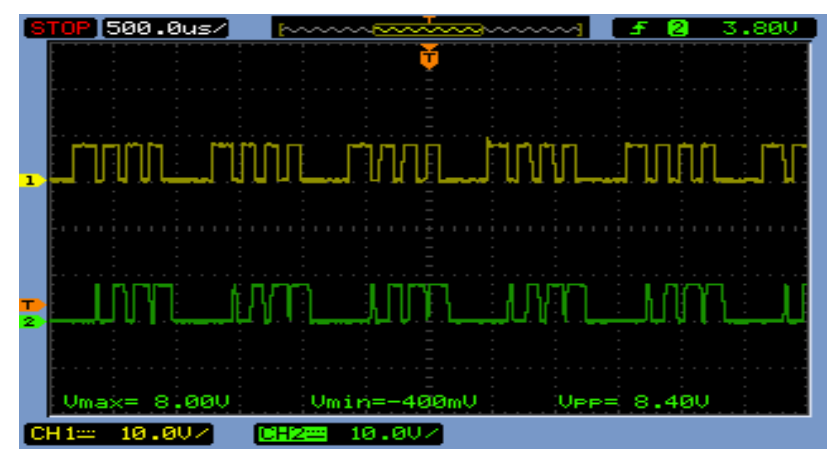

Fig.13a.Time-domain waveform of the input and output signal

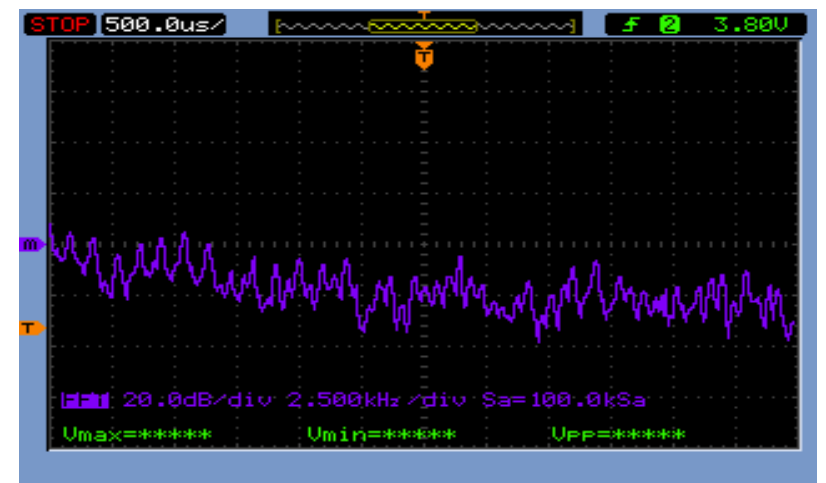

Fig.13b.Spectrum of the input signal

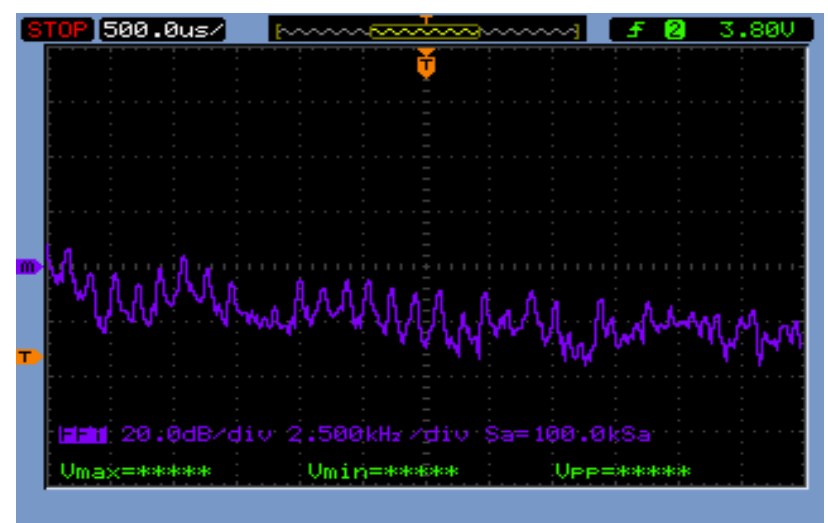

Fig.13c.Spectrum of the output signal

The OOK signals are transmitted using white LED to convert the electrical signal into corresponding optical signal. Fig.7a and Fig.7b represents the time-domain waveform and spectrum of the input signal. The optical signal travels through the free space and solarpanel ${ }_{1}$ collects the LED signal and the surrounding ambient light. Solar panel $_{1}$ converts the detected LED signal and the surrounding ambient light into corresponding voltage. Fig.8a and Fig.8b.shows the time-domain waveform and spectrum of the solar panel $l_{1}$ signal. In an indoor environment considered, the ambient light noise sources are from indirect sunlight and fluorescent light lamps operated by conventional ballast. The indirect sunlight induces strong direct current (DC) and the rectified sine wave form is emitted from the fluorescent light lamp operated by conventional ballast. Thus ambient light noise reduction technique becomes essential to attenuate the ambient light noise.

Hence another solar panel $_{2}$ is used on the opposite of solar panel $_{1}$ at an angle of $180^{\circ}$ to track only the ambient light. The arrangement must be such that, both the solar panels track the equal amount of ambient light then only ambient light noise reduction is practically possible. The two solar panels are tested to detect equal amount of ambient light by switching off the transmitted LED signal. In the electronics lab of B.S.Abdur Rahman university where the experimentation is carried out solar panel $1_{1}$ tracks more ambient light than solar panel $\mathrm{l}_{2}$. Hence a voltage source $\mathrm{V}_{\mathrm{s}}$ is added to the output of solarpanel ${ }_{2}$ to make both the solar panels track equal ambient light noise. Then, the experimentation was carried out by switching on the transmitted signal through LED. Fig.9a and Fig.9b shows the time domain waveform and spectrum of the solar panel ${ }_{2}$ signal. Difference amplifier (DA) helps to mitigate the ambient light noise and restore back the received signal. Fig.10a and Fig.10b shows the time domain waveform and spectrum of the difference amplifier (DA) signal.

The signal received from difference amplifier (DA) stage is very weak in the order of $\mathrm{mV}(320 \mathrm{mV})$. Hence voltage amplifier is used to amplify the $320 \mathrm{mV}$ signal to $6 \mathrm{~V}$ signal. Fig.11a and Fig.11b shows the time-domain waveform and spectrum of voltage amplifier signal. Voltage comparator is used to convert the amplified signal into the corresponding digital signal. Thus digital signal of $8.40 \mathrm{~V}$ amplitude is obtained similar to the transmitted signal. Fig.12a and Fig. $12 \mathrm{~b}$ shows the time-domain waveform and spectrum of voltage comparator signal. Thus the transmitted signal is retrieved back at the receiver with the attenuation of ambient light noise in the voltage comparator stage. Fig.13a, Fig.13b, Fig. $13 \mathrm{c}$ shows the time-domain waveform and spectrum of 
input signal and the output signal. The signal to noise ratio is calculated using the given formula given by eq (9)

$\operatorname{SNR}(\mathrm{dB})=20 \log _{10} \mathrm{~V}_{\mathrm{s}} / \mathrm{V}_{\mathrm{n}}$

Where $\mathrm{V}_{\mathrm{s}}$ denotes the signal voltage at the solarpanel1 when the LED is on, while $\mathrm{V}_{\mathrm{n}}$ denotes the measured noise voltage when the LED is off. Table 1 shows the measured SNR value by using the proposed noise reduction technique.

Table. 1. Measured SNR using the proposed noise reduction technique

\begin{tabular}{l|l|l}
\hline & \multicolumn{1}{|c|}{$\begin{array}{c}\text { Single Solar } \\
\text { panel }\end{array}$} & $\begin{array}{c}\text { Orthogonal solar } \\
\text { panels and } \\
\text { difference } \\
\text { amplifier }\end{array}$ \\
\hline $\begin{array}{l}\text { Signal } \\
\text { voltage(mV) }\end{array}$ & 320 & 290 \\
$\begin{array}{l}\text { Noise } \\
\text { voltage(mV) } \\
\begin{array}{l}\text { Signal-to-noise } \\
\text { ratio (dB) }\end{array}\end{array}$ & 120 & 30 \\
\hline
\end{tabular}

From table 1, it can be observed the SNR value decreases when using the single solar panel at the receiver and significantly increases using the proposed noise reduction technique.

The experimental setup of indoor VLC system using the proposed receiver is depicted in Fig. 14.

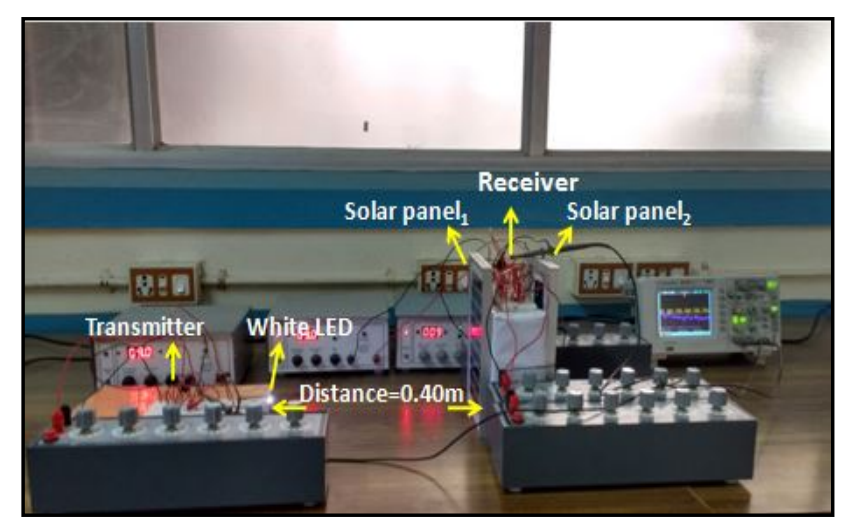

Fig.14.Experimental indoor VLC system using the proposed receiver to attenuate ambient light noise

The characteristics curve of output voltage generated at solarpanel $_{1}$ receiver for different distances is shown in Fig.15.

In Fig.15, it can be observed that the output voltage decreases with respect to increase in distance between the transmitter and receiver.

The characteristic analysis of the transmission distance and the LED optical power is presented in Fig.16. The experimentation is carried out at usual room light condition. It is clear that the optical power of LED reduces with respect to the transmission distance.

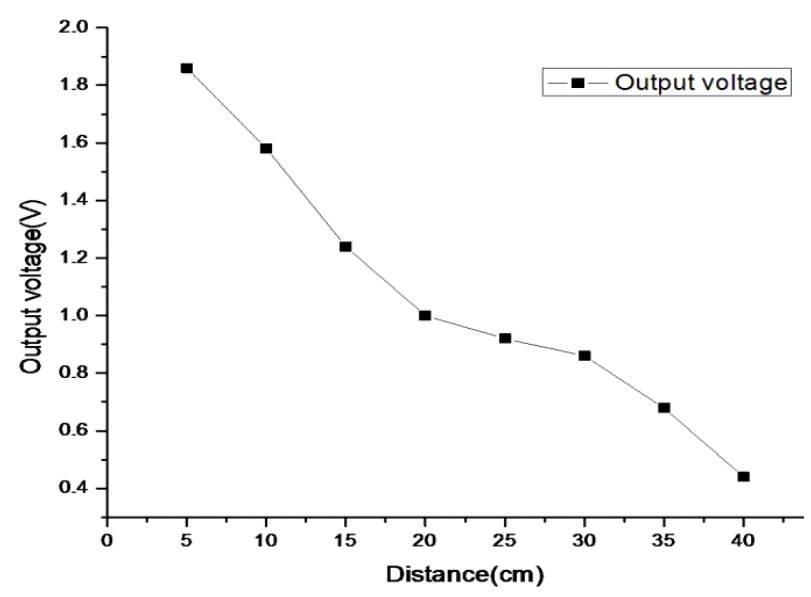

Fig.15. Output voltage generated at solarpanel ${ }_{1}$ receiver at different distances

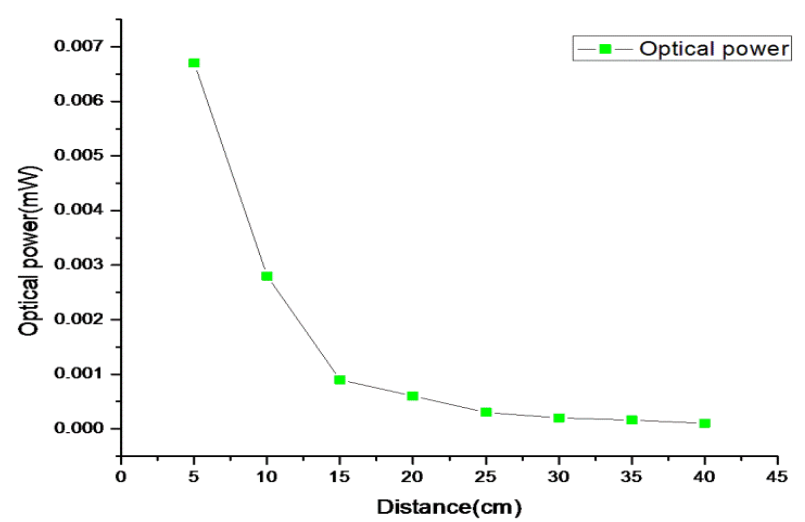

Fig.16.Optical power with respect to communication distance

Transmission distance of $0.40 \mathrm{~m}$ is achieved in this experimentation. This can be further improved by using reflector and magnifying lens. Increase in data rates can be achieved by using different LEDs, solar panel of better efficiency or appropriate modulation scheme.

\section{Conclusion}

This paper presents a new VLC receiver design to reduce ambient light noise. The design is based on two solar panels placed opposite to each other at an angle of $180^{\circ}$ and the arrangement must be such that it tracks equal amount of ambient light. The difference amplifier is used in the next stage to mitigate the ambient light noise. Experimental results show that the proposed receiver enhance the SNR performance of indoor VLC system.

This is an Open Access article distributed under the terms of the Creative Commons Attribution Licence 


\section{References}

1. W.A.Cahyadi, Y.H.Kim, Y.H.Chung and C.Ahn, "Mobile phone camera-based indoor visible light communications with rotation compensation", IEEE photonics journal, vol.8.no.2, 2016.

2. S.Wang, F.Chen, L.Liang, S.He, Y.Wang, X.Chen and W.Lu, "A high performance blue filter for a white-LED-based visible light communication system", IEEE wireless communications, vol.22.no.2, pp.61-67, 2016.

3. C.Rohner, S.Raza, D.Puccinelli and T.Voigt, "Security in visible light communication: novel challenges and opportunities", Sensors \& Transducers, vol.192, no.9, pp.9-15, 2015.

4. P.H.Pathak, X.Feng, P.Hu and P.Mohapatra, "Visible light communication, networking and sensing: A survey, potential and challenges", IEEE communications surveys and tutorials.vol.17.no.4.pp.2047-2077,2015

5. T.Zhang, S.Guo and H. Chen, "Enhancing the bit error rate of visible light communication systems using channel estimation and channel coding", wireless communications, networking and applications, vol.348, pp.51-58, 2015.

6. C.H.Yeh, C.H.Chow, H.Y.Chen, Y.L.Liu and D.Z.Hsu, "Investigation of phosphor-LED lamp for real time half-duplex wireless VLC system", Journal of optics, vol.18, no.6,pp.1$6,2016$.

7. M.Saadi, L.Wattisuttikulkij, Y.Zhao and P.Sangwongngam, "Visible Light Communication: Opportunities, Challenges and Channel Models", International Journal of Electronics and Informatics, 2012.

8. D.Karunatilaka, F.Zafar, V.Kalavally, R.Parthiban, "LED based indoor visible light communications: state of the art", IEEE communications surveys and tutorials.vol.17.no.3.pp.1649-1677, 2015.

9. Z.Wang, D.Tsonev, S.Videv and H.Haas, "On the Design of a Solar-panel Receiver for Optical Wireless Communications with Simultaneous Energy Harvesting" ,IEEE Journal on Selected Areas in Communications, vol. 33,no.8,2015

10.Z.Wang, D.Tsonev, S.Videv and H.Haas, "Towards Self-powered Solar Panel Receiver for Optical Wireless Communication" IEEE ICC-Optical Networks and Systems, 2014

11. S.Kim, J.Won and S.Nahm, "Simultaneous reception of solar power and visible light communication using a solar cell", Optical Engineering, vol.53, 2014.

12. S. Zhang, D. Tsonev, S. Videv ,S. Ghosh ,G. A. Turnbull, D. W. Samuel and H.Haas, "Organic solar cells as high-speed data detectors for visible light communication" ,optica,vol.2,no.7,2015

13. B.Malik and X.Zhang, "Solar panel receiver system implementation for visible light communication", ICECS conference, pp.502-503, 2015.

14. H.Y.Chen, K.Liang, C.Y.Chen, S.H.Chen and C.W.Chow, "Passive optical receiver for visible light communication (VLC)"IEEE conference-TENCON, pp.1-2, 2015.

15. C.Carrascal, I.Demirkol and J.Paradells, "On-Demand Sensor Node Wake-Up Using Solar Panels and Visible Light Communication", Sensors, vol.16, no.418, pp.1-19, 2016.

16. Y.Liu, H.Chen, K.Liang, C.W.Hsu, C.W.Chow and C.H.Yeh, "Visible light communication using receivers of camera image sensor and solar cell", IEEE photonics journal, vol.8, no.1, 2016. 